\title{
Process Automation System Development of Lyocell Staple Fiber Plant Based on PlantPAx
}

\author{
Yongwei $\mathrm{Li}^{1, \mathrm{a}}$, Kuan $\mathrm{Yu}^{2, \mathrm{~b}}$, Bo Zhü ${ }^{3, \mathrm{c}}$, Ronghuan $\mathrm{Han}^{1, \mathrm{~d}}$, \\ Yubo $\mathrm{Li}^{1, \mathrm{e}}$, Jianlong Wang ${ }^{1, \mathrm{f}}$ \\ ${ }^{1}$ Shandong Yingli Industrial Co., Ltd, P.R.China \\ ${ }^{2}$ School of Material Science and Engineering, Shandong Jianzhu University, P.R.China \\ ${ }^{3}$ School of Material Science and Engineering, Shandong University, P.R.China \\ a liyongwei@sdonly.com, b13605314535@139.com, ' zhubo@sdu.edu.cn, \\ d hanronghuan@sdonly.com, ${ }^{\mathrm{e}}$ liyubo@sdonly.com, ${ }^{\mathrm{f}}$ wangjianlong@sdonly.com,
}

Keywords: lyocell staple fiber, process automation system, Ethernet/IP, PlantPAx

Abstract: A process automation system based on PlantPAx platform was developed for a lyocell staple fiber plant with annual capacity of 15000 tons. The system has realized simple operation and optimization of the plant, full-automatic startup and shutdown of the plant units, control and diagnostics of malfunctions, logging and documentation of all operation sequences and process data. With the application of distributed architecture, redundant technology and Ethernet/IP industrial Ethernet, it has shown high performance and high reliability during the pilot production in 2015 .

\section{Introduction}

Lyocell is a form of rayon which consists of regenerated cellulose fiber made from dissolving pulp using dry jet-wet spinning. Since there is little waste product during the manufacturing process, lyocell is called the Green Fiber of $21^{\text {st }}$ century.

A lyocell staple fiber plant with annual capacity of 15000 tons has been building in Shandong, China since 2013. There are more than 5000 control points in the plant, as listed in Table 1.

Table 1. Control points list

\begin{tabular}{c|c|c|c|c}
\hline & Analog Input & Analog Output & Digital Input & Digital Output \\
\hline Instruments \& Valves & 720 & 244 & 841 & 499 \\
\hline Motors(Direct Start) & 0 & 0 & 2167 & 580 \\
\hline Inverters & \multicolumn{4}{|c}{404}
\end{tabular}

So an autonomous, high-performance process automation system must be designed to achieve the required automation degree.

\section{System Architecture}

The process automation system was designed based on Rockwell Automation's PlantPAx platform. It has a modular multi-layer structure to ensure the performance, scalability and reliability, as shown in Fig. 1. 


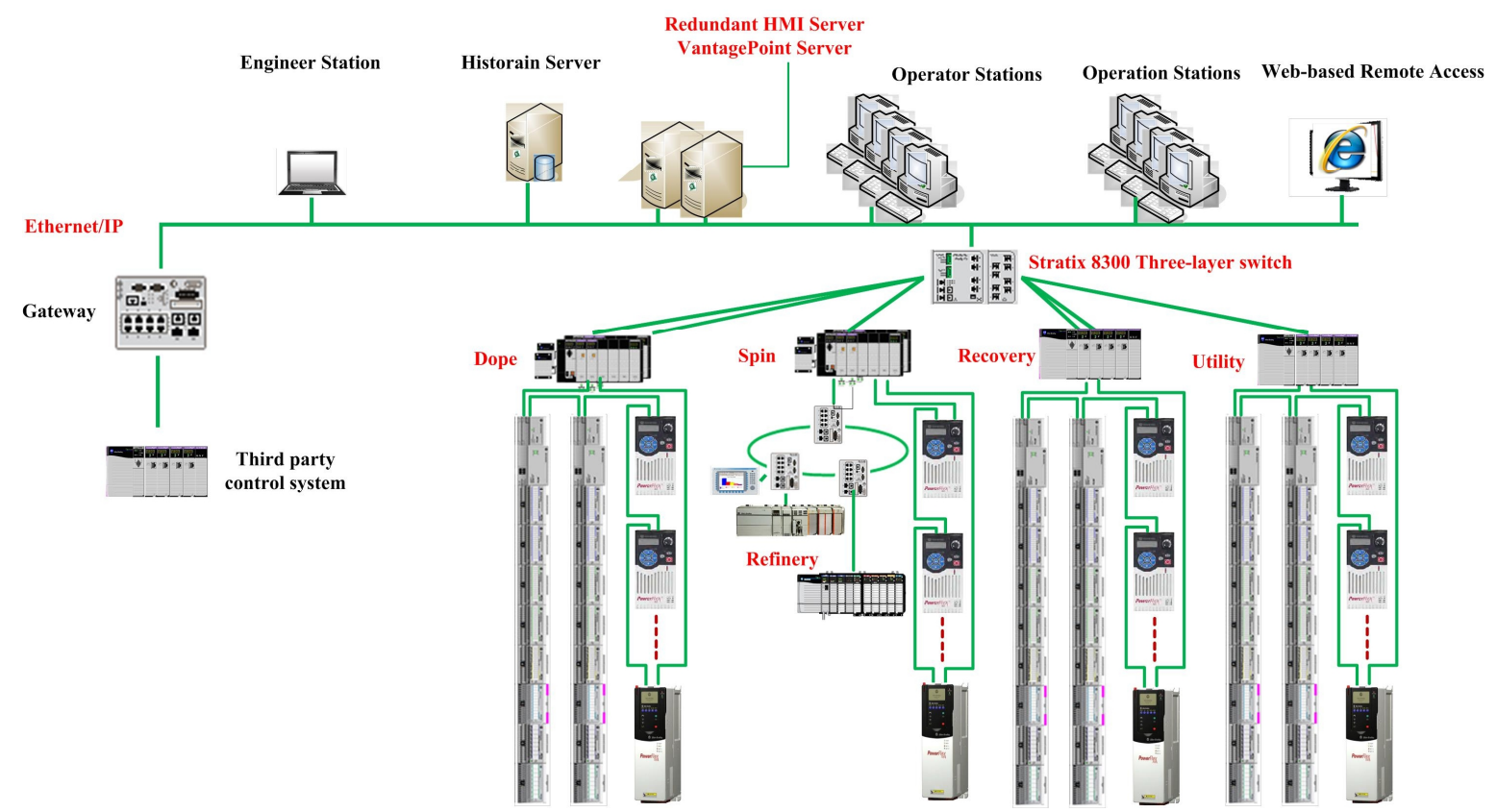

Fig.1 System architecture

The management level consists of six operator stations (computers with FactoryTalkView SE Client installed), two redundant HMI servers (servers with FactoryTalkView SE Server installed), one historian server (server with Historian Server and Vantagepoint Server installed) and two engineer stations (laptops with FactoryTalkView Studio and RSLogix5000 installed) ${ }^{[1]}$.

The process level is divided into five groups according to the process flow, including:

Dope: process area 10-20, cellulose pre-treatment and dope preparation.

Spin: process area 30-40, spin, take-up, tower washing and cutting.

Refinery: process area 50-60, after-treatment, cross-linking and drying.

Recovery: process area 70-80, purification and evaporation.

Utility: process area 90-110, auxiliary process.

Each group has one or more ControlLogix5571 program controller as the process kernel. Distributed 1794 Flex I/O modules which provide Removal and Insertion under Power and module level diagnostics have been used for the I/O level.

Compared with traditional Profibus-DP, Ethernet/IP has faster speed, better anti-interference ability and lower cost. Compared with other industrial Ethernet, Ethernet/IP realizes real-time control without modifying standard TCP/IP protocol. So we choose Ethernet/IP as the bus system between management level, process level and I/O level.

Any third party control system that doesn't support Ethernet/IP can communicate with the process automation system via gateway.

Various redundant technologies have been adopted in this plan to realize high reliability, including redundant controllers, redundant HMI Servers, Ethernet/IP DLR (Device Level Ring), REP (Resilient Ethernet Protocol) etc.

\section{Ethernet/IP Network Configuration}

Since Ethernet/IP has been adopted as the system bus and field bus, all the operator stations, servers, program controllers, 1794 Flex I/O adapters and inverters exchange data with each other via Ethernet/IP.

The overall Ethernet/IP network configuration is shown in Fig. 2. 


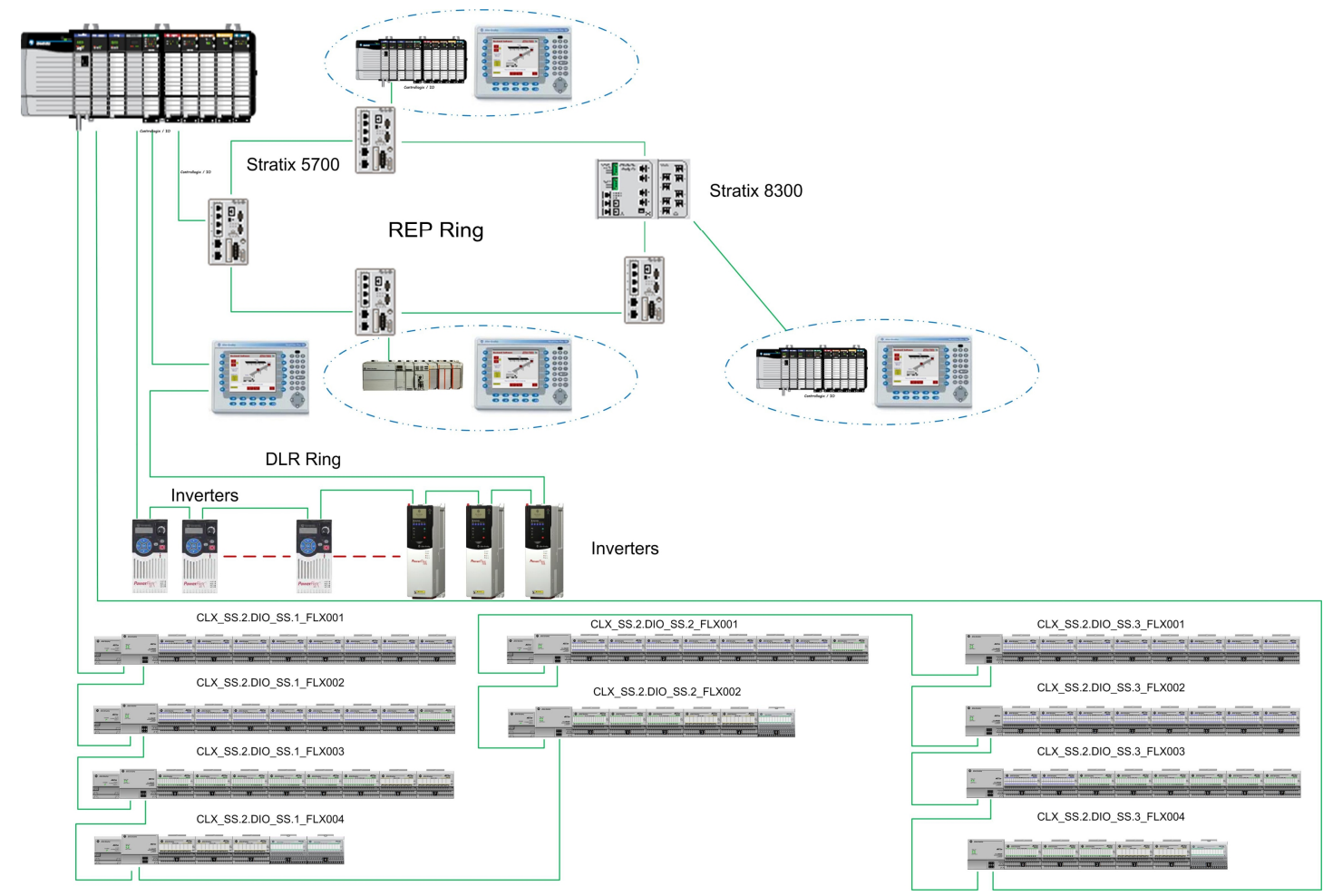

Fig. 2 Ethernet/IP network configuration

The plant has more than 400 Ethernet/IP devices, so we choose a Stratix 8300 three-layer switch with 26 ports as the core of network management, and construct a REP ring between the Stratix 8300 switch and four Stratix 5700 switches. The Stratix 8300 was configured as VTP Server, all the Stratix 5700 were configured as VTP clients, and the whole network was divided into three VLAN (192.168.123.x/255.255.255.0, 192.168.1.x/255.255.255.0, 192.168.0.x/255.255.255.0) $)^{[2]}$. Each Ethernet/IP device is connected to corresponding VLAN port according to device type or supplier.

DLR rings are constructed between the program controllers, 1794 Flex I/O adapters and inverters equipped with dual-port adapter to provide reliable communication redundancy.

\section{Software Development}

Visualization Software Development. Visualization software was developed using Rockwell FactoryTalkView Studio, taking the PIDs (Pipe \& Instrument Diagram) as the template, so that process engineer can master the software operation quickly. There are 78 operation pictures in total, and cellulose pre-treatment operation is shown in Fig. 3.

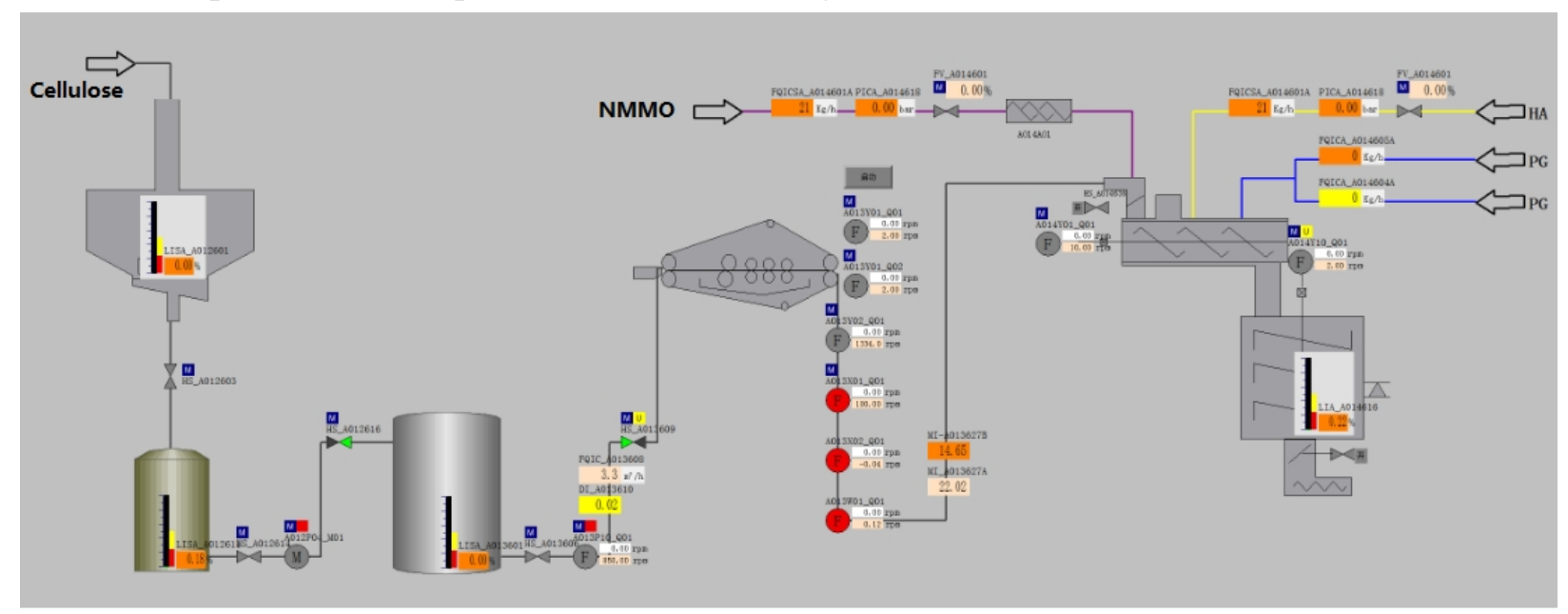

Fig. 3 Cellulose pre-treatment 
Program Controller Software Development. Program controller software was developed using Rockwell RSLogix5000. The key of program controller software includes inverter speed control, analog signal control and sequence control.

Inverter Speed Control. Powerflex series inverter can be inserted into an RSLogix5000 project as a new hardware module, and related tags will be automatically generated ${ }^{[3]}$. By assignment to a tag or reading tag value, we can get the inverter status and control inverter speed.

For take-up machine and tower-washing machine, torque control is preferred. If the active torque is equal or larger than torque settings, the inverter speed will automatically reduce to avoid machinery damage.

Enhanced PID Control. Enhanced PID (PIDE) Control is mainly used for analog signal control, such as temperature, pressure and liquid level. The PIDE uses a velocity form algorithm of the PID equation that the loop works on change in error to change the output. The velocity form algorithm is much easier to apply for more advanced applications such as adaptive gains or multi-loop selection ${ }^{[4-5]}$. This algorithm is shown as Eq. 1.

$$
\mathrm{C} V_{n}=\mathrm{C} V_{n-1}+K_{p} \Delta E+K_{i} E \Delta t+K_{D} \frac{E_{n}-2 E_{n-1}+E_{n-2}}{\Delta t}
$$

Where:

$\mathrm{CV}=$ Controlled Variable

\section{$\mathrm{E}=$ Error}

$\Delta t=$ Update time

$\mathrm{Kp}=$ Proportional Gain

$\mathrm{Ki}=$ Integral gain

$\mathrm{Kd}=$ Derivative gain

The Enhanced PID also supports cascade/ratio control, as shown in Fig. 4.

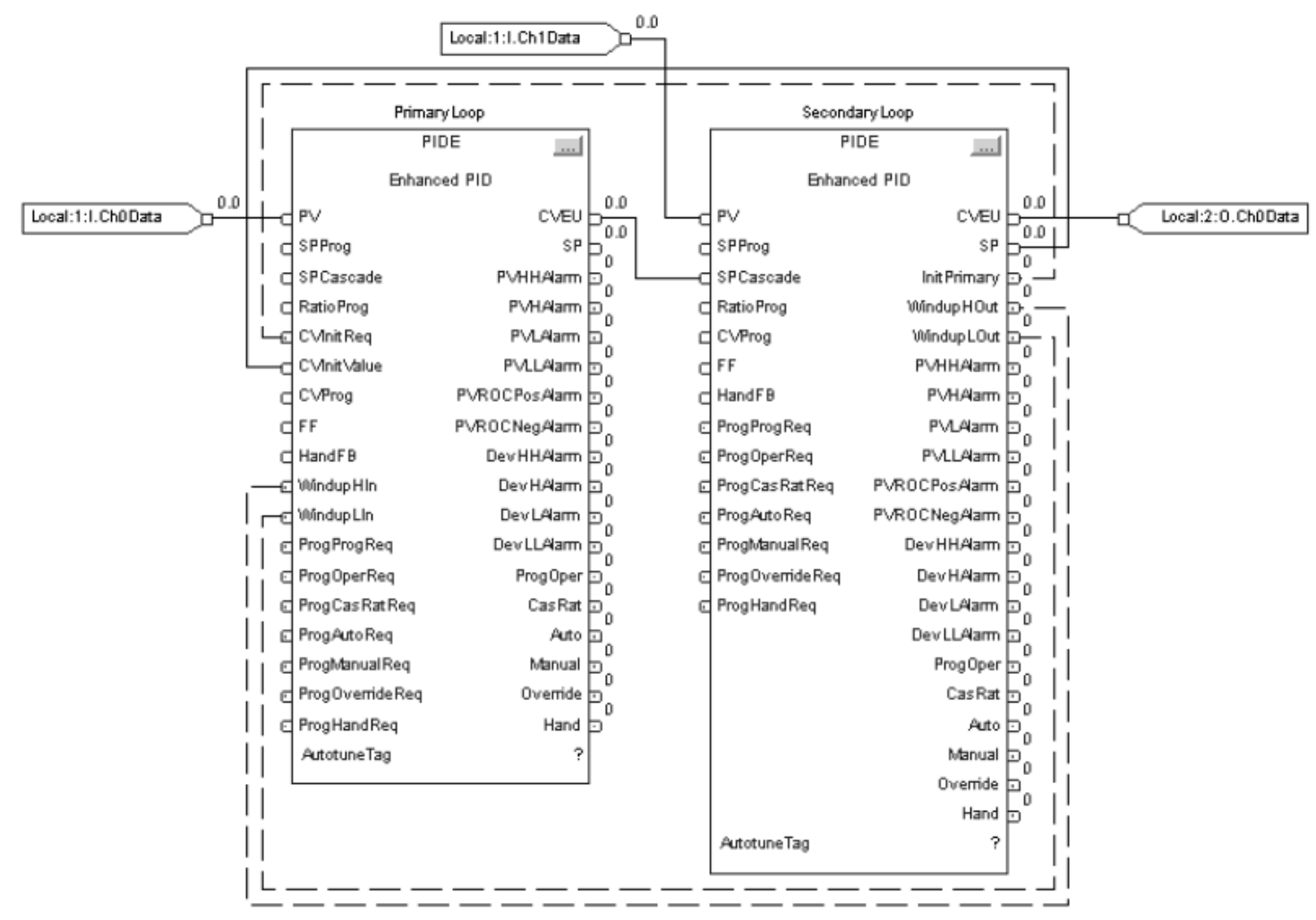

Fig. 4 PIDE cascade/ratio control 
Sequence Control. Sequence control is applied in the following process area, such as process water preparation, pulper, enzyme preparation and pulp dewatering. We realized sequence control by using Rockwell Automation Sequencer Object (P_Seq) ${ }^{[4]}$, as shown in Fig. 5.

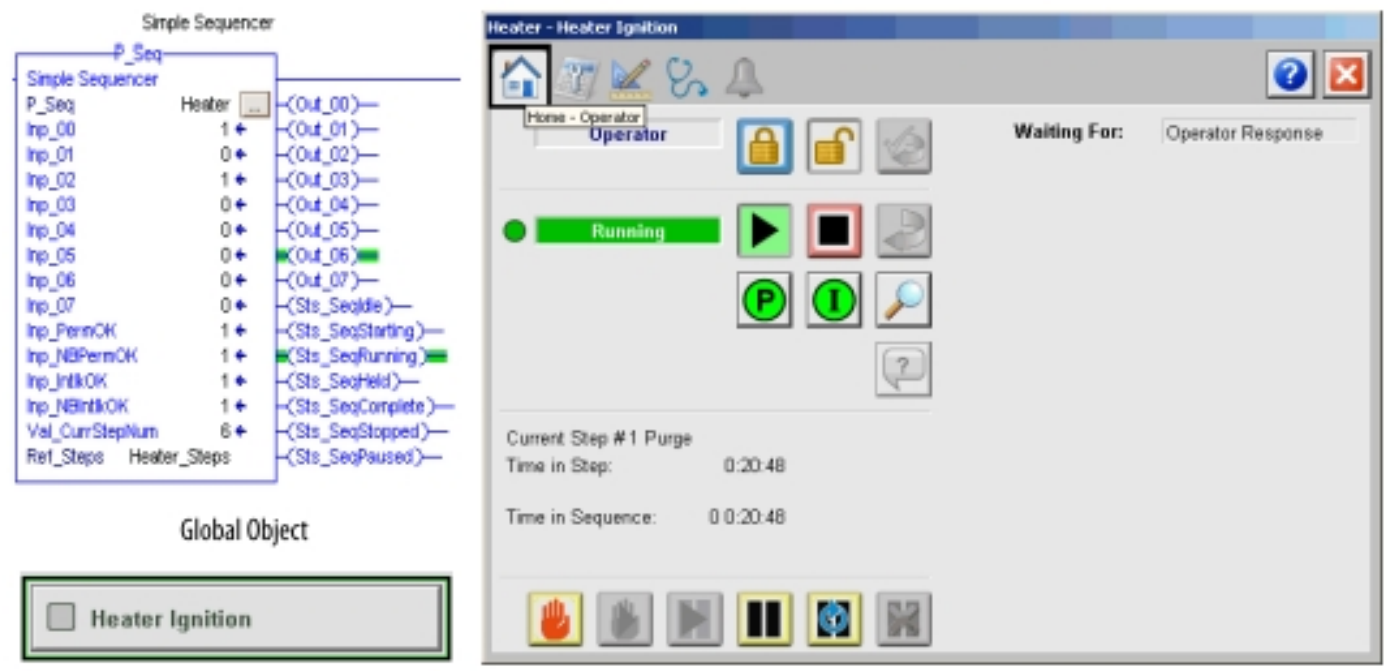

Fig.5 Sequence control

PlantPAx platform provides perfect and powerful library for advanced process control, so some traditional complex problem (such as sequence control, modular multivariable control) can be solved easily.

\section{Conclusions}

The process automation system has been put into operation since March 2015. The pilot production has shown that:

(1)This design has shown high performance, reliability and scalability.

(2)This system realized simple operation and optimization of the plant, full-automatic startup and shutdown of the plant units, control and diagnostics of malfunctions, logging and documentation of all operation sequences and process data.

(3)PlantPAx platform is powerful and easy to use, and can shorten development cycle of process automation system.

\section{References}

[1] PlantPAx Reference Manual [M]. Rockwell Automation, 2012.2.

[2] Stratix 8000 and 8300 Ethernet Managed Switches [M]. Rockwell Automation, 2012.12.

[3] Logix5000 Controllers General Instructions Reference Manual [M]. Rockwell Automation, 2009.10.

[4] Logix5000 Controllers Advanced Process Control and Drives Instructions [M]. Rockwell Automation, 2012.11.

[5] Using the PIDE Instruction [M]. Rockwell Automation, 2005.8. 